\title{
Article \\ Physical and Mental Health Related Quality of Life and Their Influencing Factors on Sexual Minority Women in Korea
}

\author{
Ssirai Kim ${ }^{1,2} \mathbb{D}$ and Smi Choi-Kwon $1,3, *$ \\ 1 College of Nursing, Seoul National University, Seoul 03080, Korea; ssirai@snu.ac.kr \\ 2 Department of Healthcare Administration, Seoul National University Hospital, Seoul 03080, Korea \\ 3 The Research Institute of Nursing Science, Seoul National University, Seoul 03080, Korea \\ * Correspondence: smi@snu.ac.kr; Tel.: +82-2-740-8830
}

check for

updates

Citation: Kim, S.; Choi-Kwon, S. Physical and Mental Health Related Quality of Life and Their Influencing Factors on Sexual Minority Women in Korea. Int. J. Environ. Res. Public Health 2021, 18, 2115. https:// doi.org/10.3390/ijerph18042115

Academic Editors: Tinashe Dune, Zelalem Mengesha, Pranee Liamputtong and Amit Arora

Received: 19 January 2021

Accepted: 18 February 2021

Published: 22 February 2021

Publisher's Note: MDPI stays neutral with regard to jurisdictional claims in published maps and institutional affiliations.

Copyright: (C) 2021 by the authors. Licensee MDPI, Basel, Switzerland. This article is an open access article distributed under the terms and conditions of the Creative Commons Attribution (CC BY) license (https:// creativecommons.org/licenses/by/ $4.0 /)$.

\begin{abstract}
Korean sexual minority women (SMW) often experience discrimination, but their healthrelated quality of life (HRQoL) remains to be investigated. Therefore, we aimed to assess the levels of mental and physical HRQoL of Korean SMW and their influencing factors using data from the Korean Sexual Minority Women's Health Study (2017) in a cross-sectional study, which included lesbian and bisexual females $\left(N=736\right.$; age $\geq 19$ years). The HRQoL was measured using SF-36v2 ${ }^{\circledR}$; moreover, separate multiple linear regression analyses were conducted to identify the factors influencing mental and physical HRQoL. The physical and mental HRQoL scores were average (52.38 \pm 7.65) and low $(38.33 \pm 12.64)$, respectively. Significant factors influencing the physical HRQoL were bisexuality, minority stress, perceived social support, and physical activity. The same factors-apart from physical activity-were associated with mental HRQoL. Therefore, to improve the HRQoL of SMW, it is necessary to lower their minority stress and increase social support. Moreover, special attention is needed regarding bisexual women in Korea.
\end{abstract}

Keywords: mental health; physical health; sexual minority women; health-related quality of life; Korea

\section{Introduction}

Health inequities among sexual minority women (SMW) are well-established. Compared with heterosexual women, SMW experience worse mental health outcomes [1-5], including anxiety and depression, and have poor physical health $[1,6,7]$, including highrisk health-related lifestyle factors [8,9] such as smoking and alcohol abuse. Furthermore, SMW's health-related quality of life (HRQoL) is lower than their heterosexual counterparts [10-15].

The low health level of SMW may be due to minority stress, the unique and additive stress experienced by people with minority identities [5,16]. According to Minority Stress Theory [5,17], stress from stigmatized identity status or stigmatizing societal structure negatively affects health. Previous studies [3,5] reported that distal stress, such as violence or victimization against sexual identity, was related to the poor mental health of sexual minority people. Proximal stress-vigilance or internalized homophobia also related to an individual's perceptions and predicted adverse health outcomes [3,4]. On the other hand, in the Psychological Mediation Framework, stress could result in maladaptive coping and emotion regulation that increases the risk of poor mental health $[16,18,19]$. Moreover, when exposed to stressors, fear of disclosing identities prevents sexual minority people from feeling sufficiently supported [16]. However, sexual minorities are not only passive victims of stress; their identity can also be a protective factor [17]. One of the most positive effects on the health of SMW is social support $[1,2,20]$. Community connectivity was also addressed as another positive factor affecting mental health [20]. Furthermore, factors that affect mental health also appear to affect physical health $[1,6,21]$. Specifically, high minority stress, low physical activity, and various health-related lifestyle factors are related to low physical HRQoL [21]. Another important point when describing the health of SMW 
is the difference between inner groups. In previous studies, bisexual women reported poorer health than lesbians $[1,3,15,22,23]$. However, most of these findings were reported in Western countries [11-15,21].

The expression of hatred toward sexual minorities has emerged as an important social issue in Korea [24,25]. Christianity, the religion that more than a third of Koreans belong to, shows strong opposition to sexual minorities, more so than other religions [26]. In addition, a traditional family system that values heterosexuality, and deep-rooted Confucian virtues, are hallmarks of the anti-LGBT atmosphere in Korea [25,27-29]. These societal concerns pose a threat to the health of sexual minorities. Previous studies showed a higher prevalence of depressive symptoms and suicidal ideation among sexual minorities than the general population in Korea [30,31]. Depressive symptoms were related to discrimination against sexual orientation or lower community connectedness [31]. On the other hand, family support was a significant factor that impacted life satisfaction among Korean sexual minorities [32].

A previous study highlighted the need to research the health of lesbians and bisexual women since they are relatively ignored in Korean sexual minority health studies [30]. Past studies have explored the quality of life or life satisfaction in Korean sexual minorities, but participants were limited to gay men or lesbians only $[32,33]$. Few studies examined the comprehensive health status of Korean SMW including bisexuals.

Therefore, we attempted to examine the level of physical and mental HRQoL in Korean SMW and identify the factors associated with each HRQoL. Our main hypotheses were as follows: (1) SMW in Korea may have a low level of HRQoL, (2) high minority stress level lowers the physical and mental HRQoL of SMW in Korea, (3) high perceived social support positively affect physical and mental HRQoL, and (4) Korean bisexual women have lower HRQoL than lesbians do.

\section{Materials and Methods}

\subsection{Study Design and Participants}

This cross-sectional study used data from the "Korean Sexual Minority Women's Health Study", which was conducted to examine Korean SMW's HRQoL and health behaviors including health service utilization. The data were collected via an online survey form using SurveyMonkey, a survey hosting site, from September to November 2017. The participants of the study included Korean women who were 19 years or older, lesbian, bisexual, or belonged to other sexual minorities. Gender minorities were excluded from the study. We recruited the participants through social media, LGBT organizations' newsletters, or online LGBT communities including those targeted towards SMW over 30 years old. Participants were informed about the study's purpose and design on the study webpage. Among the 1256 respondents who answered questions in the survey, only 801 fully completed the survey. Further, we excluded those who chose "asexual", "uncertain", or "other" for the sexual identity question $(N=65)$. In total, $736 \mathrm{SMW}$ were selected.

\subsection{Study Variables}

\subsubsection{Sociodemographic Variables and Sexual Identity}

Sociodemographic characteristics included age (groups: 19-29, 30-39, and 40-51), city of residence (capital city-Seoul, or other cities), education level (high school or pre-college and graduate), employment status (student, employed, or unemployed), and annual family income level. Annual family income was divided into $<$ USD 50,000 and $\geq$ USD 50,000 based on the average income per Korean household at the time of survey-1 USD equaled approximately $1000 \mathrm{KRW}$ [34]. Participants who described themselves as lesbian, bisexual, or pansexual were included in this study. We categorized them into two groups: lesbian and bisexual (including pansexuals). 


\subsubsection{Health-Related Lifestyle Factors}

We examined the following health-related lifestyle factors: smoking (non-smokers and smokers, who answered "Yes" to the question "Are you currently a smoker"), highrisk drinking (two drinks at a time twice weekly), and physical activity [35]. Physical activity was measured using the Korean version of the International Physical Activity Questionnaire (IPAQ) [36], and then categorized as low, moderate, and high activity.

\subsubsection{Minority Stress}

To measure day-to-day minority stress, we used the Daily Heterosexist Experience Questionnaire (DHEQ) developed by Balsam et al. [37] with permission from the original author. The measure was translated into Korean through reverse translation. Out of the original nine subgroups (50 items), we used seven - vigilance, discrimination and harassment, gender expression, victimization, family of origin, vicarious trauma, and isolation. We excluded parenting and HIV subscales in the current study because of the legal and social context. Under Korean law, same-sex marriages are not allowed, and single parent adoptions are rarely implemented. Moreover, the prevalence of HIV among Korean women is much lower than among men [38]. Participants responded to "How much has this problem distressed or bothered you during the past 12 months?" by choosing from the options ranging from 0 (did not happen/not applicable to me) to 5 (it happened, and I was extremely bothered) on a six-point Likert scale. Responses were later recoded so that both " 0 " and " 1 " represented " 1 " (did not bother me). The rest of the responses remained the same. Subsequently, we computed a mean score from the responses to all items, reflecting the mean level of distress a participant feels related to these experiences. Higher scores indicated higher stress related to experiencing, and being bothered by, instances of heterosexism. We used 35 items for this study after factor analysis. At the time of development, Cronbach's $\alpha$ of each subgroup ranged from 0.72 to 0.87 [37], and the overall Cronbach's $\alpha$ was 0.855 in this study.

\subsubsection{Perceived Social Support}

The Multidimensional Scale of Perceived Social Support (MSPSS)—available for free from http:/ /gzimet.wixsite.com/mspss (accessed on 30 May 2017)—was used to measure the perceived social support from three sources: significant other, family, and friends [39]. The scale consists of 12 items with response options presented on a seven-point Likert-type scale, ranging from 1 (very strongly disagree) to 7 (very strongly agree). Items included statements such as "There is a special person who is around when I am in need," and "My family really tries to help me." The total MSPSS score was calculated by adding the scores of all 12 items and dividing the total score by 12. Each domain score was obtained in the same way by using 4 as the divisor. Higher MSPSS scores reflected higher perceived social support. Cronbach's $\alpha$ of the MSPSS was 0.91 at the time of development and 0.893 in this study.

\subsubsection{Physical and Mental Health-Related Quality of Life}

To measure HRQoL, we used a standardized SF-36v2 ${ }^{\circledR}$ Korean version by Optum (License no.: QM041038), consisting of 36 items and eight domains. The domains were physical functioning, bodily pain, role physical, and general health, all included in the physical component summary (PCS) calculation; and mental health, social functioning, role emotional, and vitality, included in the calculation of the mental component summary (MCS). In our study, MCS indicates mental-health-related quality of life (Mental HRQoL) and PCS indicates physical-health-related quality of life (Physical HRQoL)

By using QualityMetric software (QualityMetric Incorporated, Lincoln, RI, USA), we converted the data from the SF-36v2 ${ }^{\circledR}$ to a numerical score using norm-based scoring, normalized so that each domain's mean score was $50(S T D \pm 10)$ for the general population. Mean scores below 47 and above 53 indicated poorer and better health status, respectively, than the normal range [40]. 


\subsection{Statistical Analysis}

The number of samples for this study was set based on a significance level of 0.05 , a medium effect size of 0.15 , a power of 0.95 , and a total of 12 variables required for regression analysis. As a result of the analysis using $G^{*}$ power 3.1 software [41], the minimum number of samples was 184, indicating that this study satisfies the appropriate number of samples. We used complete case analysis to deal with missing data, and only those who reported complete data were included in the analysis. We also checked outliers for entry errors and used all 736 completed data for final analysis.

First, to examine the HRQoL and explanatory variables of Korean SMW, we used descriptive statistics and conducted bivariate analysis. Age was calculated as median and inter-quartile ranges. Categorical variables are presented as counts and proportions, and continuous variables are presented as mean and standardized deviation $(S D)$. We performed factor analysis and calculated Cronbach's $\alpha$. The participants' physical and mental HRQoL were compared according to their characteristics, using t-test or ANOVA. Moreover, post hoc $F$ tests were conducted to detect potential differences among more than three groups. The correlation between the continuous variables and HRQoL was explored through Pearson's correlation analysis. Second, separate multiple linear regression analyses were performed to determine factors influencing physical and mental HRQoL. To check whether the assumptions of regression analysis were satisfied, tolerance, variation inflation factor, and Durbin-Watson were calculated for each model. In the first model (Model 1), we examined the association between sexual identity and HRQoL variables, controlling for sociodemographic variables. We added smoking, high-risk drinking, and physical activity level in the second model (Model 2). Minority stress was included in Model 3. In the last model (Model 4), perceived social support was added. All statistical analyses were conducted using IBM SPSS Statistics for Windows, Version 25.0 (IBM Corp, Armonk, NY, USA), except the scores of HRQoL, which were initially calculated using QualityMetric (QualityMetric Incorporated, Lincoln, RI, USA) software. $p$-values were based on a twosided significance level of 0.05 .

\subsection{Ethics Declarations}

We obtained informed consent from the study participants via an online survey form. As a reward for their participation, we provided two options: an online coupon equivalent to $\$ 5$ if subjects left their contact details, or a $\$ 5$ donation to LGBT organizations if they wanted to keep their identity private. The Korean Sexual Minority Women's Health Study was approved by the institutional review board of the Seoul National University (IRB No. $1707 / 002-005)$.

\section{Results}

\subsection{Health-Related Quality of Life by Participants' Characteristics}

The median age of the Korean SMW participants was 25, and 50.8\% were lesbian. Of the total participant group, $23 \%$ were current smokers and $11 \%$ showed high-risk drinking. Furthermore, $75 \%$ had been engaged in moderate to high physical activity, and the mean physical HRQoL of all 736 participants was $52.38 \pm 7.65$. Interestingly, the physical HRQoL of the participants showed significant differences in terms of education level $(p=0.017)$, employment status $(p=0.012)$, sexual identity $(p=0.026)$, smoking $(p=0.031)$, and physical activity $(p<0.0001)$, Table 1 .

The mean of the mental HRQoL of all 736 participants was $38.33 \pm 12.64$. The mental HRQoL of the participating SMWs showed significant differences in terms of age group $(p<0.0001)$, annual family income $(p=0.018)$, employment status $(p<0.0001)$, and sexual identity $(p=0.001)$.

The mean minority stress $(2.14 \pm 0.48)$ was negatively correlated with both physical $(p<0.0001)$ and mental $(p<0.0001)$ HRQoL. In contrast, the mean perceived social support $(4.89 \pm 1.11)$ showed a positive correlation with both physical $(p=0.001)$ and mental $(p<0.0001)$ HRQoL. 
Table 1. Health-Related Quality of Life by Participants' Characteristics.

\begin{tabular}{|c|c|c|c|c|c|c|c|c|c|c|}
\hline & \multirow{2}{*}{\multicolumn{2}{|c|}{ Total }} & \multicolumn{8}{|c|}{ Health-Related Quality of Life } \\
\hline & & & \multicolumn{4}{|c|}{ Physical (PCS) } & \multicolumn{4}{|c|}{ Mental (MCS) } \\
\hline & $\mathbf{N}$ or $\mathbf{M}$ & $\%$ or SD & $\mathbf{M}$ & SD & $\begin{array}{c}t / F \text { or } r \\
\text { (Scheffe) }\end{array}$ & $p$-Value & $\mathbf{M}$ & SD & $\begin{array}{c}t / F \text { or } r \\
\text { (Scheffe) }\end{array}$ & $p$-Value \\
\hline Total & 736 & 100 & 52.38 & 7.65 & & & 38.33 & 12.64 & & \\
\hline Age group & & & & & & & & & & \\
\hline $19-29$ (a) & 559 & 76.0 & 52.59 & 7.81 & & & 37.44 & 12.56 & 7.877 & $<0.0001$ \\
\hline $30-39(b)$ & 146 & 19.8 & 51.59 & 6.94 & 0.998 & 0.369 & 40.28 & 12.96 & $(\mathrm{a}<\mathrm{c})$ & \\
\hline $40-51(\mathrm{c})$ & 31 & 4.2 & 52.27 & 7.91 & & & 45.22 & 9.09 & & \\
\hline Median (Q1-Q3) & \multicolumn{2}{|c|}{$25(21-29)$} & & & & & & & & \\
\hline \multicolumn{11}{|l|}{ City } \\
\hline Capital city & 344 & 46.7 & 52.29 & 7.36 & -0.275 & 0.783 & 38.55 & 12.39 & 0.445 & 0.656 \\
\hline Other & 392 & 53.3 & 52.45 & 7.90 & & & 38.14 & 12.86 & & \\
\hline \multicolumn{11}{|l|}{ Education } \\
\hline $\begin{array}{l}\text { High school or } \\
\text { under }\end{array}$ & 70 & 9.5 & 50.30 & 7.41 & 2.396 & 0.017 & 35.71 & 13.80 & 1.684 & 0.096 \\
\hline Above college & 666 & 90.5 & 52.59 & 7.64 & & & 38.61 & 12.49 & & \\
\hline \multicolumn{11}{|l|}{$\begin{array}{l}\text { Annual family } \\
\text { income }\end{array}$} \\
\hline$<$ USD 50,000 & 525 & 71.3 & 52.04 & 7.66 & -1.858 & 0.064 & 37.66 & 12.93 & -2.372 & 0.018 \\
\hline$\geq \operatorname{USD} 50,000$ & 211 & 28.7 & 53.20 & 7.57 & & & 40.00 & 11.74 & & \\
\hline \multicolumn{11}{|l|}{ Employment } \\
\hline Student(a) & 349 & 47.4 & 53.14 & 7.81 & 4.413 & & 36.93 & 12.81 & 14.268 & $<0.0001$ \\
\hline Employed(b) & 325 & 44.2 & 51.44 & 7.20 & $(\mathrm{~b}<\mathrm{a})$ & 0.012 & 40.83 & 11.52 & $(a, c<b)$ & \\
\hline Unemployed(c) & 62 & 8.4 & 53.01 & 8.54 & & & 33.14 & 14.51 & & \\
\hline \multicolumn{11}{|l|}{ Sexual Identity } \\
\hline Lesbian & 374 & 50.8 & 52.99 & 7.68 & 2.233 & 0.026 & 39.90 & 12.52 & 3.436 & 0.001 \\
\hline Bisexual & 362 & 49.2 & 51.74 & 7.57 & & & 36.72 & 12.56 & & \\
\hline \multicolumn{11}{|l|}{ Smoking } \\
\hline Yes & 173 & 23.51 & 51.28 & 7.79 & 2.161 & 0.031 & 37.54 & 12.81 & 0.947 & 0.344 \\
\hline No & 563 & 76.49 & 52.71 & 7.58 & & & 38.58 & 12.58 & & \\
\hline \multicolumn{11}{|l|}{$\begin{array}{l}\text { High -risk } \\
\text { drinking }\end{array}$} \\
\hline Yes & 85 & 11.54 & 51.452 & 8.0581 & 1.185 & 0.236 & 39.281 & 13.9093 & -0.736 & 0.462 \\
\hline No & 651 & 88.46 & 52.496 & 7.5897 & & & 38.208 & 12.4657 & & \\
\hline \multicolumn{11}{|l|}{ Physical Activity } \\
\hline High (a) & 249 & 33.83 & 53.17 & 7.06 & 8.552 & & 39.34 & 12.22 & & \\
\hline Moderate (b) & 305 & 41.44 & 52.92 & 7.60 & $(c<a, b)$ & $<0.0001$ & 38.36 & 12.32 & 1.953 & 0.143 \\
\hline Low (c) & 182 & 24.73 & 50.37 & 8.17 & & & 36.91 & 13.60 & & \\
\hline Minority Stress & 2.14 & 0.48 & & & -0.151 & $<0.0001$ & & & -0.238 & $<0.0001$ \\
\hline $\begin{array}{l}\text { Perceived Social } \\
\text { Support }\end{array}$ & 4.89 & 1.11 & & & 0.124 & 0.001 & & & 0.428 & $<0.0001$ \\
\hline
\end{tabular}

PCS: Physical Component Summary, MCS: Mental Component Summary; N: Number; M: Mean; SD: Standard Deviation.

\subsection{Subdomains of Minority Stress and Perceived Social Support}

We further analyzed the subdomains of minority stress and perceived social support to determine the characteristics of those variables. Among the minority stress subdomains, stress due to vicarious trauma ( $3.77 \pm 0.91)$ was the highest, followed by stress from vigilance (3.04 \pm 1.14$)$; moreover, the lowest stress level was from victimization $(1.03 \pm 0.23)$. Among the subdomains of perceived social support, the support from significant others (5.54 \pm 1.39$)$ was the highest, followed by friends ( $5.33 \pm 1.38)$. The support from family was the lowest $(3.81 \pm 1.59)$, Table 2 . 
Table 2. Subdomains of Minority Stress and Perceived Social Support $(\mathrm{N}=736)$.

\begin{tabular}{cccc}
\hline & Range & Mean & SD \\
\hline Minority Stress & $1 \sim 5$ & 2.14 & 0.48 \\
Vigilance & $1 \sim 5$ & 3.04 & 1.14 \\
Discrimination \& Harassment & $1 \sim 5$ & 1.53 & 0.76 \\
Gender expression & $1 \sim 5$ & 1.29 & 0.56 \\
Victimization & $1 \sim 5$ & 1.03 & 0.23 \\
Family of origin & $1 \sim 5$ & 1.40 & 0.68 \\
Vicarious trauma & $1 \sim 5$ & 3.77 & 0.91 \\
Isolation & $1 \sim 5$ & 2.21 & 1.11 \\
\hline Perceived Social Support & $1 \sim 7$ & 4.89 & 1.39 \\
Significant other & $1 \sim 7$ & 5.54 & 1.59 \\
Family & $1 \sim 7$ & 3.81 & 1.38 \\
\hline Friends & $1 \sim 7$ & 5.33 & \\
\hline
\end{tabular}

\subsection{Predictors of Physical Health-Related Quality of Life}

The factors affecting the physical HRQoL of our subjects are presented in Table 3. There is no multi-collinearity problem between the independent variables. In Model 1, employment status and sexual identity had a statistically significant influence on physical HRQoL, explaining the physical HRQoL variance of $2.1 \%(p=0.003)$. In Model 2, employment status, sexual identity, and physical activity were statistically significant factors influencing physical HRQoL. The explanatory power of Model 2 increased to $4.2 \%$ $(p<0.0001)$. In Model 3, employment status, sexual identity, physical activity, and minority stress were statistically significant factors influencing physical HRQoL. The explanatory power of Model 3 increased to 5.5\% $(p<0.0001)$. In the final model (Model 4$)$, employment status $(p=0.03)$, sexual identity $(p<0.001)$, physical activity $(p<0.001)$, minority stress $(p=0.003)$, and perceived social support $(p=0.020)$ were significant factors influencing physical HRQoL. These results confirmed this study's hypothesis. The explanatory power of Model 4 was $6.1 \%(p<0.0001)$.

Table 3. Predictors on Physical Health-related Quality of Life $(N=736)$.

\begin{tabular}{|c|c|c|c|c|c|c|c|c|c|c|c|c|c|c|c|c|c|}
\hline \multirow{2}{*}{\multicolumn{2}{|c|}{ Variables }} & \multicolumn{4}{|c|}{ Model 1} & \multicolumn{4}{|c|}{ Model 2} & \multicolumn{4}{|c|}{ Model 3} & \multicolumn{4}{|c|}{ Model 4} \\
\hline & & B & $\beta$ & $t$ & $p$ & B & $\beta$ & $t$ & $p$ & B & $\beta$ & $\mathbf{t}$ & $p$ & B & $\beta$ & $\mathbf{t}$ & $p$ \\
\hline (Constants) & & 52.00 & & 72.61 & $<0.0001$ & 53.22 & & 61.93 & $<0.0001$ & 57.55 & & 37.00 & $<0.0001$ & 54.17 & & 25.42 & $<0.0001$ \\
\hline \multirow[t]{3}{*}{ Age } & 19-29(ref) & & & & & & & & & & & & & & & & \\
\hline & 30-39 & -0.52 & -0.03 & -0.65 & 0.52 & -0.24 & -0.01 & -0.30 & 0.77 & -0.51 & -0.03 & -0.64 & 0.52 & -0.60 & -0.03 & -0.75 & 0.45 \\
\hline & $40-51$ & 0.19 & 0.00 & 0.13 & 0.90 & 0.64 & 0.02 & 0.44 & 0.66 & -0.03 & 0.00 & -0.02 & 0.98 & -0.37 & -0.01 & -0.25 & 0.80 \\
\hline City & Capital city & 0.37 & 0.02 & 0.66 & 0.51 & 0.42 & 0.03 & 0.74 & 0.46 & 0.42 & 0.03 & 0.73 & 0.46 & 0.45 & 0.03 & 0.79 & 0.43 \\
\hline Education & $\begin{array}{l}\text { Above } \\
\text { College }\end{array}$ & -1.81 & -0.07 & -1.84 & 0.07 & -1.61 & -0.06 & -1.65 & 0.10 & -1.65 & -0.06 & -1.70 & 0.09 & -1.35 & -0.05 & -1.38 & 0.17 \\
\hline Income & $\geq \$ 50,000$ & 1.01 & 0.06 & 1.63 & 0.10 & 0.81 & 0.05 & 1.32 & 0.19 & 0.89 & 0.05 & 1.45 & 0.15 & 0.76 & 0.04 & 1.24 & 0.22 \\
\hline \multirow[t]{3}{*}{ Employment } & Employed(ref) & & & & & & & & & & & & & & & & \\
\hline & Student & 1.64 & 0.11 & 2.39 & 0.02 & 1.47 & 0.10 & 2.16 & 0.03 & 1.47 & 0.10 & 2.17 & 0.03 & 1.43 & 0.09 & 2.12 & 0.03 \\
\hline & Unemployed & 1.71 & 0.06 & 1.60 & 0.11 & 1.80 & 0.07 & 1.69 & 0.09 & 1.85 & 0.07 & 1.75 & 0.08 & 1.97 & 0.07 & 1.86 & 0.06 \\
\hline \multirow{3}{*}{$\begin{array}{c}\text { Sexual } \\
\text { Identity } \\
\text { Smoking } \\
\text { High-risk } \\
\text { Drinking }\end{array}$} & Bisexual & -1.56 & -0.10 & -2.69 & 0.01 & -1.72 & -0.11 & -2.98 & $<0.001$ & -1.92 & -0.13 & -3.34 & $<0.001$ & -1.93 & -0.13 & -3.36 & $<0.001$ \\
\hline & Yes & & & & & -1.16 & -0.06 & -1.70 & 0.09 & -1.12 & -0.06 & -1.66 & 0.10 & -1.12 & -0.06 & -1.66 & 0.10 \\
\hline & Yes & & & & & -0.55 & -0.02 & -0.62 & 0.54 & -0.60 & -0.02 & -0.68 & 0.50 & -0.69 & -0.03 & -0.78 & 0.43 \\
\hline \multirow{3}{*}{$\begin{array}{l}\text { Physical } \\
\text { Activity }\end{array}$} & High(ref) & & & & & & & & & & & & & & & & \\
\hline & Medium & & & & & -0.31 & -0.02 & -0.48 & 0.63 & -0.26 & -0.02 & -0.41 & 0.68 & -0.19 & -0.01 & -0.29 & 0.77 \\
\hline & Low & & & & & -2.70 & -0.15 & -3.67 & $<0.001$ & -2.69 & -0.15 & -3.68 & $<0.001$ & -2.48 & -0.14 & -3.38 & $<0.001$ \\
\hline \multirow{2}{*}{\multicolumn{2}{|c|}{$\begin{array}{c}\text { Minority Stress } \\
\text { Perceived Social Support }\end{array}$}} & & & & & & & & & -1.96 & -0.12 & -3.33 & $<0.001$ & -1.76 & -0.11 & -2.98 & 0.003 \\
\hline & & & & & & & & & & & & & & 0.60 & 0.09 & 2.32 & 0.02 \\
\hline \multicolumn{2}{|c|}{$\mathrm{R}^{2}$} & \multicolumn{4}{|c|}{0.032} & \multicolumn{4}{|c|}{0.057} & \multicolumn{4}{|c|}{0.072} & \multicolumn{4}{|c|}{0.079} \\
\hline \multirow{2}{*}{\multicolumn{2}{|c|}{$\begin{array}{c}\text { Adjusted } R^{2} \\
F\end{array}$}} & \multicolumn{4}{|c|}{0.021} & \multicolumn{4}{|c|}{0.042} & \multirow{2}{*}{\multicolumn{4}{|c|}{$\begin{array}{l}0.055 \\
4.291\end{array}$}} & \multicolumn{4}{|c|}{0.061} \\
\hline & & \multirow{2}{*}{\multicolumn{4}{|c|}{$\begin{array}{l}2.999 \\
0.003\end{array}$}} & \multicolumn{4}{|c|}{$\begin{array}{c}3.671 \\
<0.0001\end{array}$} & \multicolumn{3}{|c|}{4.291} & & \multicolumn{4}{|c|}{4.392} \\
\hline \multicolumn{2}{|c|}{ p } & & & & & \multicolumn{3}{|c|}{$<0.0001$} & & & $<0.0$ & & & & $<0.0$ & 001 & \\
\hline
\end{tabular}

ref. = reference. Model 1: Age group, City, Education level, Annual family income, Employment status, Sexual identity. Model 2: Age group, City, Education level, Annual family income, Employment status, Sexual identity, Smoking, Physical activity. Model 3: Age group, City, Education level, Annual family income, Employment status, Sexual identity, Smoking, Physical activity, Minority stress. Model 4: Age group, City, Education level, Annual family income, Employment status, Sexual identity, Smoking, Physical activity, Minority stress, Perceived social support. 


\subsection{Predictors of Mental-Health-Related Quality of Life}

The factors affecting the mental HRQoL of our participants are presented in Table 4. There is no multicollinearity problem between independent variables. In Model 1, age, education level, annual family income, employment status, and sexual identity had a statistically significant influence on mental HRQoL, explaining mental HRQoL variance of $5.3 \%(p<0.0001)$. In Model 2 , age, employment status, sexual identity, and physical activity were statistically significant factors influencing mental HRQoL. The explanatory power of Model 2 increased to 6.0\% $(p<0.0001)$. In Model 3, annual family income, employment status, sexual identity, physical activity, and minority stress were statistically significant factors influencing mental HRQoL. The explanatory power of Model 3 increased to $11.3 \%$ $(p<0.0001)$. In the final model (Model 4$)$, employment status $(p<0.0001)$, sexual identity $(p<0.0001)$, minority stress $(p<0.0001)$, and perceived social support $(p<0.0001)$ were significant factors impacting mental HRQoL. This result confirmed the study's hypothesis. The explanatory power of Model 4 was $24.7 \%(p<0.0001)$.

Table 4. Predictors on Mental Health-related Quality of Life $(N=736)$.

\begin{tabular}{|c|c|c|c|c|c|c|c|c|c|c|c|c|c|c|c|c|c|}
\hline \multirow{2}{*}{\multicolumn{2}{|c|}{ Variables }} & \multicolumn{4}{|c|}{ Model 1} & \multicolumn{4}{|c|}{ Model 2} & \multicolumn{4}{|c|}{ Model 3} & \multicolumn{4}{|c|}{ Model 4} \\
\hline & & B & $\beta$ & $t$ & $p$ & B & $\beta$ & $t$ & $p$ & B & $\beta$ & $\mathbf{t}$ & $p$ & B & $\beta$ & $\mathbf{t}$ & $p$ \\
\hline \multicolumn{2}{|c|}{ (Constants) } & 46.66 & & 34.93 & \multicolumn{2}{|c|}{$<0.000142 .54$} & & 230.25 & \multicolumn{2}{|c|}{$<0.000156 .38$} & & 22.64 & \multicolumn{2}{|c|}{$<0.000131 .704$} & & 10.06 & $<0.0001$ \\
\hline \multirow[t]{3}{*}{ Age } & 19-29(ref) & & & & & & & & & & & & & & & & \\
\hline & $30-39$ & 0.37 & 0.01 & 0.28 & 0.78 & 0.69 & 0.02 & 0.53 & 0.60 & -0.19 & -0.01 & -0.15 & 0.88 & -0.82 & -0.03 & -0.69 & 0.49 \\
\hline & $40-51$ & 4.93 & 0.08 & 2.06 & 0.04 & 5.36 & 0.09 & 2.25 & 0.03 & 3.21 & 0.05 & 1.37 & 0.17 & 0.73 & 0.01 & 0.34 & 0.74 \\
\hline City & Capital city & 0.57 & 0.02 & 0.61 & 0.54 & 0.51 & 0.02 & 0.55 & 0.58 & 0.49 & 0.02 & 0.54 & 0.59 & 0.72 & 0.03 & 0.87 & 0.39 \\
\hline Education & $\begin{array}{l}\text { Above } \\
\text { College }\end{array}$ & -3.02 & -0.07 & -1.89 & 0.06 & -2.83 & -0.07 & -1.77 & 0.08 & -2.95 & -0.07 & -1.90 & 0.06 & -0.73 & -0.02 & -0.50 & 0.62 \\
\hline Income & $\geq \$ 50,000$ & 1.95 & 0.07 & 1.93 & 0.05 & 1.72 & 0.06 & 1.71 & 0.09 & 1.96 & 0.07 & 2.00 & 0.05 & 1.03 & 0.04 & 1.14 & 0.26 \\
\hline \multirow[t]{3}{*}{ Employment } & Employed(ref) & & & & & & & & & & & & & & & & \\
\hline & Student & -3.23 & -0.13 & -2.89 & $<0.000$ & -3.36 & -0.13 & -3.02 & $<0.001$ & -3.37 & -0.13 & -3.11 & $<0.001$ & -3.63 & -0.14 & -3.64 & $<0.0001$ \\
\hline & Unemployed & -6.94 & -0.15 & -3.99 & $<0.000$ & -7.06 & -0.16 & -4.04 & $<0.0001$ & -6.88 & -0.15 & -4.05 & $<0.001$ & -6.05 & -0.13 & -3.87 & $<0.0001$ \\
\hline \multirow{3}{*}{$\begin{array}{l}\text { Sexual } \\
\text { Identity } \\
\text { Smoking } \\
\text { High-risk } \\
\text { Drinking }\end{array}$} & Bisexual & -2.16 & -0.09 & -2.29 & 0.02 & -2.33 & -0.09 & -2.47 & 0.01 & -2.99 & -0.12 & -3.24 & $<0.001$ & -3.04 & -0.12 & -3.57 & $<0.0001$ \\
\hline & Yes & & & & & -2.01 & -0.07 & -1.80 & 0.07 & -1.89 & -0.06 & -1.74 & 0.08 & -1.87 & -0.06 & -1.87 & 0.06 \\
\hline & Yes & & & & & 0.83 & 0.02 & 0.57 & 0.57 & 0.66 & 0.02 & 0.47 & 0.64 & 0.02 & 0.00 & 0.01 & 0.99 \\
\hline \multirow{3}{*}{$\begin{array}{l}\text { Physical } \\
\text { Activity }\end{array}$} & High(ref) & & & & & & & & & & & & & & & & \\
\hline & Medium & & & & & -1.52 & -0.06 & -1.44 & 0.15 & -1.37 & -0.05 & -1.34 & 0.18 & -0.82 & -0.03 & -0.87 & 0.38 \\
\hline & Low & & & & & -2.94 & -0.10 & -2.44 & 0.02 & -2.90 & -0.10 & -2.48 & 0.01 & -1.38 & -0.05 & -1.27 & 0.20 \\
\hline \multirow{2}{*}{\multicolumn{2}{|c|}{$\begin{array}{c}\text { Minority Stress } \\
\text { Perceived Social Support }\end{array}$}} & & & & & & & & & -6.24 & -0.24 & -6.65 & $<0.0001$ & -4.81 & -0.18 & -5.50 & $<0.0001$ \\
\hline & & & & & & & & & & & & & & 4.35 & 0.38 & 11.41 & $<0.0001$ \\
\hline \multicolumn{2}{|c|}{$R^{2}$} & \multicolumn{4}{|c|}{0.063} & \multicolumn{4}{|c|}{0.075} & \multicolumn{4}{|c|}{0.128} & \multicolumn{4}{|c|}{0.262} \\
\hline \multirow{2}{*}{\multicolumn{2}{|c|}{$\underset{F}{\text { Adjusted } R^{2}}$}} & \multicolumn{4}{|c|}{0.053} & \multicolumn{4}{|c|}{0.060} & \multicolumn{4}{|c|}{0.113} & \multicolumn{4}{|c|}{0.250} \\
\hline & & \multirow{2}{*}{\multicolumn{4}{|c|}{$\begin{array}{c}6.147 \\
<0.0001\end{array}$}} & \multicolumn{4}{|c|}{4.889} & \multicolumn{4}{|c|}{8.180} & & 18.2 & 262 & \\
\hline \multicolumn{2}{|c|}{$\begin{array}{l}\mathrm{F} \\
p\end{array}$} & & & & & & $<0.00$ & 001 & & & $<0.00$ & & & & $<0.0$ & 001 & \\
\hline
\end{tabular}

ref. = reference. Model 1: Age group, City, Education level, Annual family income, Employment status, Sexual identity Model 2: Age group, City, Education level, Annual family income, Employment status, Sexual identity, Smoking, Physical Activity model 3: Age group, City, Education level, Annual family income, Employment status, Sexual identity, Smoking, Physical activity, Minority stress Model 4: Age group, City, Education level, Annual family income, Employment status, Sexual identity, Smoking, Physical activity, Minority stress, Perceived social support.

\section{Discussion}

To the best of our knowledge, this is the first study to examine HRQoL and its influencing factors among SMW in Korea. We found that the mental HRQoL of Korean SMW participants was low with a score level of $38.33 \pm 12.64$, whereas, the physical HRQoL score was $52.38 \pm$ 7.65 , which is in the average range. Interestingly, minority stress, perceived social support, and sexual identity affected both the physical and mental HRQoL of the SMW, but physical activity was related only to their physical HRQoL. Furthermore, their mental HRQoL was very low, to the point of clinically impaired functioning [40]. The mental HRQoL was even lower than that of other Korean groups measured with the same tool, including people with fatigue (45.4 47.8) or patients with liver diseases (46.2 \pm 11.7$)$ [42-44]. Our finding is similar to other studies that revealed a lower HRQoL among sexual minorities than the general 
population $[10-12,22,45,46]$. In terms of international comparison, the mental HRQoL score in this study was lower than that of the lesbians in some countries overseas $(49.10 \pm 10.10)$ or transgender (41.60 \pm 12.76$)$ groups [46,47].

The low mental HRQoL may, in part, be due to minority stress, which was confirmed as a risk factor during the regression analysis. These results were similar to previous studies $[1,2,12,15,48]$. However, our participants' minority stress type was different: they rarely reported stress from events marked by direct discrimination (including being physically assaulted) due to sexual orientation. In contrast, some previous studies reported the experience of direct victimization $[37,49]$. Furthermore, we found that vicarious trauma in our subjects was high. In particular, the item analysis showed the highest level of stress from vicarious trauma, especially due to experiences such as "Hearing politicians say negative things about LGBT people" or "Hearing about LGBT people you know/do not know being treated unfairly" [37]. As this study was conducted in 2017, it could be assumed that the stress of Korean SMW was elevated when they encountered negative remarks about homosexuality during the Korean presidential election campaign [50]. Moreover, some previous studies pointed out the role of distal stress [3,49] when, for example, frequent exposure to negative media messages about sexual minorities were associated with greater psychological distress [2]. The results of our study showed that even if discrimination was not experienced physically, hearing that others were being discriminated against could impact mental health in the same way as experiencing it firsthand.

Furthermore, our subjects experienced high stress due to vigilance, which is related to proximal stress. In hiding their identities, Korean SMW experience increased stress. In previous studies, chronic experiences of discrimination and rejection were reported to increase vigilance [16]. Stress from stigma induced self-monitoring and vigilant concealing of one's stigmatized identity. Korean SMW could have developed a coping mechanism to conceal their identity in general situations to avoid discrimination. In other words, poor mental health could have been caused by not only experiencing direct discrimination but also changing the coping and emotion-regulation process [16]. However, since our study did not assess the direct relationship among stressors, general psychological processes, and psychopathology, further research is needed in the future.

On the other hand, high social support was related to higher mental HRQoL among Korean SMW participants. Perceived social support not only positively affected mental HRQoL, but was the most influential factor. Similarly, in previous studies, the social support factor protected the mental health and quality of life of sexual minorities [13,51,52]. When we examined the sources of social support, Korean SMW received a high level of social support from significant others and friends. It seems that Korean SMW are actively forming positive relationships in the chosen relationship.

However, the level of social support in our study was somewhat lower than that found in previous studies [1,53]. In particular, we found that family social support was the lowest. These results have important implications for family-oriented Korean society. In previous Korean studies, family solidarity had a significant effect on the quality of life of sexual minorities, and family support was a significant factor in their life satisfaction [32,33]. Low family support could be the consequence of exposure to stigma-related stress, which may lead to concealment of self-identity to avoid rejection [16]. In this study, "I can talk about my problems with my family" showed the lowest result in item analysis. It was significantly lower than items like "My family really tries to help me". This result may be indirectly related to the fear of revealing sexual orientation. However, future research is needed to see the relationship between stressors and lower supports. Moreover, in our study, groups with low family support often had low annual family income or education levels. The low socio-economic status (SES) itself may have hampered Korean SMW from receiving sufficient family support. Therefore, additional attention is needed to enhance support for Korean SMW who experience low family support.

In our study, bisexual identity also explained the low mental HRQoL of Korean SMW. Similar to what was found in other studies $[1,3,15,22,23]$, bisexual women had poorer 
HRQoL than lesbians. There appear to be health disparities among Korean SMW: bisexuals may additionally experience bi-specific stress [54-58]. South Korea's LGBT community is still growing and is characterized by a well-established majority of gay and lesbian organizations. In such an environment, bisexual women may feel increasingly isolated because they do not have access to communities that meet their needs and hence do not receive the required support $[15,59]$.

Our results indicate that the physical HRQoL (52.38 \pm 7.65$)$ of Korean SMW was in the average or normal range [40]. This differs from previous studies where sexual minorities' physical HRQoL was lower than that of the general population [13] and may be related to the younger age of participants in this study. Few severe disease-related or health status cases were found among the young people in their 20-30s in the Korean general population study [60]. In our findings, physical activity increased physical HRQoL. It could be assumed that our participants had good physical HRQoL because $70 \%$ of them reported moderate or high physical activity. Surprisingly, however, we found that their smoking rate was $23.5 \%$ - much higher than the $3.5-7.5 \%$ of the general Korean female population that smokes [35,61]. Smoking was not a significant variable that directly affected physical HRQoL, but the high smoking rate suggested that they may be more likely to develop cardiovascular disease later in life [62]. Considering that both stress and smoking are risk factors for cardiovascular disease, long-term studies focused on the physical health of Korean SMW are needed [1,6,8].

Our study has the following implications for the future establishment of public health strategies. First, our study shows that structural and national intervention is required to maintain and improve the health of Korean SMW. Destigmatizing policies such as anti-discriminatory laws should be actively implemented to reduce exposure of SMW to minority stress. In addition, the government should be able to accurately identify the health status of SMW by including variables related to sexual orientation in national statistics. Furthermore, an individual-level approach is also required. Among Korean SMW in our study, bisexual women were vulnerable to poor mental health. We need to pay attention and create affirmative interventions for bisexual women. In addition, programs for those with low family support are necessary.

This study has limitations. First, since we recruited the study participants through LGBTQ organizations, it is possible that sexual minorities who were not affiliated with these groups may have been excluded. Second, to overcome the first limitation, we additionally recruited as many SMW as possible through social media. This may explain the relatively small portion of SMW over 40 years old in our study, which leads to the limitation of the generalizability for older SMW in Korea. Follow-up research, therefore, is needed at the national level and also for SMW over 40 years old. Third, there was no screening to assess whether our participants had been diagnosed with mental illness or were taking drugs, including anti-depressants. Finally, since our study is cross-sectional, it is difficult to completely infer a causality among variables even though the correlations between them were significant. Despite these limitations, we believe that this study is valuable given the findings above.

\section{Conclusions}

The mental HRQoL of Korean SMW in this study was low, and their physical HRQoL was average. Korean SMW with high minority stress or low social support showed low mental HRQoL. Additionally, Korean bisexual women had lower mental HRQoL than lesbians. In terms of the mental health of Korean SMW, it is necessary to lower minority stress and increase social support. Particular attention is needed for bisexual women. Moreover, since the level of physical activity had a significant relationship with physical HRQoL, it is necessary to develop a comprehensive health promotion program for the Korean SMW. 
Author Contributions: S.K. developed the concept of this manuscript and study protocol, collected and analyzed the data, and prepared the manuscript. S.C.-K. developed the study protocol, concept, and revised this manuscript to its final version. All authors have read and agreed to the published version of the manuscript.

Funding: This research was partially funded by Lee Chang-kuk Queer Research Fund (2017) of Beyond the Rainbow Foundation.

Institutional Review Board Statement: The study was conducted according to the guidelines of the Declaration of Helsinki, and approved by the Institutional Review Board of the Seoul National University (IRB No. 1707/002-005, 10 July 2017).

Informed Consent Statement: Informed consent was obtained from subjects involved in the study via the online survey form.

Data Availability Statement: The data are not publicly available due to privacy or ethical restrictions.

Acknowledgments: We would like to especially thank the Korean sexual minority communities for their participation and assistance in the survey development and promotion.

Conflicts of Interest: The authors declare no conflict of interest. The funders had no role in the design of the study; in the collection, analyses, or interpretation of data; in the writing of the manuscript; or in the decision to publish the results.

\section{References}

1. Ehlke, S.J.; Braitman, A.L.; Dawson, C.A.; Heron, K.E.; Lewis, R.J. Sexual minority stress and social support explain the association between sexual identity with physical and mental health problems among young lesbian and bisexual women. Sex. Roles 2020, 83, 370-381. [CrossRef]

2. Verrelli, S.; White, F.A.; Harvey, L.J.; Pulciani, M.R. Minority stress, social support, and the mental health of lesbian, gay, and bisexual Australians during the Australian Marriage Law Postal Survey. Aust. Psychol. 2019, 54, 336-346. [CrossRef]

3. Ramirez, J.L.; Paz Galupo, M. Multiple minority stress: The role of proximal and distal stress on mental health outcomes among lesbian, gay, and bisexual people of color. J. Gay Lesbian Ment. Health 2019, 23, 145-167. [CrossRef]

4. Zheng, L.; Hart, T.A.; Noor, S.W.; Wen, G. Stressors based on sexual orientation and mental health among lesbian, gay, and bisexual individuals in China: Minority stress and perceived pressure to get married. Arch. Sex. Behav 2020, 49, 1769-1782. [CrossRef] [PubMed]

5. Meyer, I.H. Prejudice, social stress, and mental health in lesbian, gay, and bisexual populations: Conceptual issues and research evidence. Psychol. Bull. 2003, 129, 674. [CrossRef] [PubMed]

6. Frost, D.M.; Lehavot, K.; Meyer, I.H. Minority stress and physical health among sexual minority individuals. J. Behav. Med. 2015, 38, 1-8. [CrossRef]

7. Eliason, M.J. Chronic physical health problems in sexual minority women: Review of the literature. Lgbt Health 2014, 1, 259-268. [CrossRef]

8. Wilson, S.M.; Gilmore, A.K.; Rhew, I.C.; Hodge, K.A.; Kaysen, D.L. Minority stress is longitudinally associated with alcohol-related problems among sexual minority women. Addict. Behav. 2016, 61, 80-83. [CrossRef]

9. Hoffman, L.; Delahanty, J.; Johnson, S.E.; Zhao, X. Sexual and gender minority cigarette smoking disparities: An analysis of 2016 Behavioral Risk Factor Surveillance System data. Prev. Med. 2018, 113, 109-115. [CrossRef] [PubMed]

10. Hutchcraft, M.L.G.; Patterson, J.G.; Teferra, A.A.; Montemorano, L.; Backes, F.J. Differences in self-reported health-related quality of life in heterosexual and sexual minority women surviving cancer: 2013 to 2018 national health interview survey. Am. Soc. Clin. Oncol. 2020, 38, e19038. [CrossRef]

11. Potter, E.C.; Patterson, C.J. Health-Related Quality of Life Among Lesbian, Gay, and Bisexual Adults: The Burden of Health Disparities in 2016 Behavioral Risk Factor Surveillance System Data. LGBT Health 2019, 6, 357-369. [CrossRef] [PubMed]

12. Yette, E.M.; Ahern, J. Health-related quality of life among black sexual minority women. Am. J. Prev. Med. 2018, 55, 281-289. [CrossRef]

13. Marti-Pastor, M.; Perez, G.; German, D.; Pont, A.; Garin, O.; Alonso, J.; Gotsens, M.; Ferrer, M. Health-related quality of life inequalities by sexual orientation: Results from the Barcelona Health Interview Survey. PLoS ONE 2018, 13, e0191334. [CrossRef] [PubMed]

14. Austin, S.B.; Gordon, A.R.; Ziyadeh, N.J.; Charlton, B.M.; Katz-Wise, S.L.; Samnaliev, M. Stigma and health-related quality of life in sexual minorities. Am. J. Prev. Med. 2017, 53, 559-566. [CrossRef]

15. Fredriksen-Goldsen, K.I.; Kim, H.-J.; Barkan, S.E.; Balsam, K.F.; Mincer, S.L. Disparities in health-related quality of life: A comparison of lesbians and bisexual women. Am. J. Public Health 2010, 100, 2255-2261. [CrossRef] [PubMed]

16. Hatzenbuehler, M.L. How does sexual minority stigma "get under the skin"? A psychological mediation framework. Psychol. Bull. 2009, 135, 707. [CrossRef] 
17. Pitoňák, M. Mental health in non-heterosexuals: Minority stress theory and related explanation frameworks review. Ment. Health Prev. 2017, 5, 63-73. [CrossRef]

18. Chang, C.J.; Fehling, K.B.; Selby, E.A. Sexual minority status and psychological risk for suicide attempt: A serial multiple mediation model of social support and emotion regulation. Front. Psychiatry 2020, 11, 385. [CrossRef]

19. Szymanski, D.M.; Dunn, T.L.; Ikizler, A.S. Multiple minority stressors and psychological distress among sexual minority women: The roles of rumination and maladaptive coping. Psychol. Sex. Orientat. Gend. Divers. 2014, 1, 412. [CrossRef]

20. Snapp, S.D.; Watson, R.J.; Russell, S.T.; Diaz, R.M.; Ryan, C. Social support networks for LGBT young adults: Low cost strategies for positive adjustment. Fam. Relat. 2015, 64, 420-430. [CrossRef]

21. Fredriksen-Goldsen, K.I.; Kim, H.-J.; Shiu, C.; Goldsen, J.; Emlet, C.A. Successful aging among LGBT older adults: Physical and mental health-related quality of life by age group. Gerontologist 2015, 55, 154-168. [CrossRef]

22. VanKim, N.A.; Erickson, D.J.; Eisenberg, M.E.; Lust, K.; Rosser, B.S.; Laska, M.N. Relationship between weight-related behavioral profiles and health outcomes by sexual orientation and gender. Obesity 2016, 24, 1572-1581. [CrossRef] [PubMed]

23. Fredriksen-Goldsen, K.I.; Shiu, C.; Bryan, A.E.; Goldsen, J.; Kim, H.-J. Health equity and aging of bisexual older adults: Pathways of risk and resilience. J. Gerontol. Ser. B Psychol. Sci. Soc. Sci. 2017, 72, 468-478. [CrossRef]

24. Hong, S.; Kim, J.; Noh, J.; Ryu, M.; Lee, S.; Lee, J. A Research on Hate-Speech in Korea and Measures for Regulation: Final Report; National Human Rights Commission of Korea: Seoul, Korea, 2016; pp. 226-228.

25. Yi, H.; Kim, S.-S. Anti-LGBT movement in South Korea and the professionals on their side. Psychol Sex. Rev. 2016, 7, 83-85.

26. Rich, T.S.; Dahmer, A.; Eliassen, I. Unpacking the roles of religion and partisanship on support for same-sex marriage: Evidence from South Korea. J. Asian Afr. Stud. 2020. [CrossRef]

27. Rich, T.S. Religion and public perceptions of gays and lesbians in South Korea. J. Homosex. 2017, 64, 606-621. [CrossRef]

28. Jung, G. Evangelical protestant women's views on homosexuality and LGBT Rights in Korea: The role of confucianism and nationalism in heteronormative ideology. J. Homosex. 2020. [CrossRef]

29. Kwak, H.W.; Kim, M.Y. Severity and Influencing Factors of Homophobia in Korean Nursing Students. Int. J. Environ. Res. Public Health 2019, 16, 4692. [CrossRef] [PubMed]

30. Yi, H.; Lee, H.; Park, J.; Choi, B.; Kim, S.-S. Health disparities between lesbian, gay, and bisexual adults and the general population in South Korea: Rainbow Connection Project I. Epidemiol. Health 2017, 39, e2017046. [CrossRef]

31. Lee, H.; Park, J.; Choi, B.; Yi, H.; Kim, S.-S. Association between discrimination and depressive symptoms among 2,162 lesbian, gay, and bisexual adults in South Korea: Does community connectedness modify the association? J. Homosex. 2021, 68, 70-87. [CrossRef] [PubMed]

32. Kang, B.C. The Effects of Perceived Social Stigma on the Life Satisfaction of Sexual Minorities. Korean J. Soc. Welf. Stud. 2011, 42, 381-417. [CrossRef]

33. Kong, S.-U.; Oh, K.-S.; No, K.-S. The comparison of the quality of life and mental health in homosexual men and heterosexual men. J. Korean Neuropsychiatr. Assoc. 2002, 41, 930-941.

34. Statistics Korea. The Survey of Household Finances and Living Conditions (SFLC) in 2017; Statistics Korea: Daejeon, Korea, 2017.

35. Korean Statistical Information Service (KOSIS). Korea National Health and Nutrition Examination Survey; KOSIS: Daejeon, Korea, 2020.

36. Oh, J.Y.; Yang, Y.J.; Kim, B.S.; Kang, J.H. Validity and Reliability of Korean Version of International Physical Activity Questionnaire (IPAQ) Short Form. J. Korean Acad. Fam. Med. 2007, 28, 532-541.

37. Balsam, K.F.; Beadnell, B.; Molina, Y. The Daily Heterosexist Experiences Questionnaire: Measuring minority stress among lesbian, gay, bisexual, and transgender adults. Meas. Eval. Couns. Dev. 2013, 46, 3-25. [CrossRef]

38. Kim, J.M.; Kim, N.J.; Choi, J.Y.; Chin, B.S. History of Acquired Immune Deficiency Syndrome in Korea. Infect. Chemother. 2020, 52, 234. [CrossRef] [PubMed]

39. Zimet, G.D.; Dahlem, N.W.; Zimet, S.G.; Farley, G.K. The multidimensional scale of perceived social support. J. Personal. Assess. 1988, 52, 30-41. [CrossRef]

40. Maruish, M.E. User's Manual for the SF-36v2 Health Survey; Quality Metric Incorporated: Lincoln, RI, USA, 2011.

41. Faul, F.; Erdfelder, E.; Buchner, A.; Lang, A.-G. Statistical power analyses using G* Power 3.1: Tests for correlation and regression analyses. Behav. Res. Methods 2009, 41, 1149-1160. [CrossRef]

42. Jang, E.S.; Kim, Y.S.; Kim, K.-A.; Lee, Y.J.; Chung, W.J.; Kim, I.H.; Lee, B.S.; Jeong, S.-H. Factors associated with health-related quality of life in Korean patients with chronic hepatitis C infection using the SF-36 and EQ-5D. Gut Liver 2018, 12, 440. [CrossRef] [PubMed]

43. Yoo, E.H.; Choi, E.S.; Cho, S.H.; Do, J.H.; Lee, S.J.; Kim, J.-H. Comparison of fatigue severity and quality of life between unexplained fatigue patients and explained fatigue patients. Korean J. Fam. Med. 2018, 39, 180. [CrossRef] [PubMed]

44. Kim, S.-H.; Jo, M.-W.; Ahn, J.; Ock, M.; Shin, S.; Park, J. Assessment of psychometric properties of the Korean SF-12 v2 in the general population. Bmc Public Health 2014, 14, 1086. [CrossRef]

45. Wong, J.Y.-H.; Choi, E.P.-H.; Lo, H.H.-M.; Wong, W.; Chio, J.H.-M.; Choi, A.W.-M.; Fong, D.Y.-T. Dating violence, quality of life and mental health in sexual minority populations: A path analysis. Qual. Life Res. 2017, 26, 959-968. [CrossRef]

46. O'Bryan, J.; Scribani, M.; Leon, K.; Tallman, N.; Wolf-Gould, C.; Wolf-Gould, C.; Gadomski, A. Health-related quality of life among transgender and gender expansive youth at a rural gender wellness clinic. Qual. Life Res. 2020, 29, 1597-1607. [CrossRef]

47. Payne, W.J. The Effect of Marriage on Lesbians' Mental Health, Physical Health, and Quality of Life; Walden University: Minneapolis, MN, USA, 2011. 
48. Shin, S. A Study on Health Related Quality of Life of Gay Men and Lesbians in Korea. Health Soc. Welf. Rev. 2013, 33, 380-416.

49. Staples, J.M.; Neilson, E.C.; Bryan, A.E.; George, W.H. The role of distal minority stress and internalized transnegativity in suicidal ideation and nonsuicidal self-injury among transgender adults. J. Sex. Res. 2018, 55, 591-603. [CrossRef] [PubMed]

50. Society of Law and Policy on Sexual Orientation and Gender Identity. Annual Review 2017: Human Rights Situation of LGBTI in South Korea (English E Korean); Korean Society of Law and Policy on Sexual Orientation and Gender Identity: Seoul, Korea, 2018.

51. Kim, H.-J.; Jen, S.; Fredriksen-Goldsen, K.I. Race/ethnicity and health-related quality of life among LGBT older adults. Gerontologist 2017, 57, S30-S39. [CrossRef] [PubMed]

52. Matthews, A.K.; Hotton, A.; Li, C.-C.; Miller, K.; Johnson, A.; Jones, K.W.; Thai, J. An internet-based study examining the factors associated with the physical and mental health quality of life of LGBT cancer survivors. LGBT Health 2016, 3, 65-73. [CrossRef] [PubMed]

53. Scandurra, C.; Bacchini, D.; Esposito, C.; Bochicchio, V.; Valerio, P.; Amodeo, A.L. The influence of minority stress, gender, and legalization of civil unions on parenting desire and intention in lesbian women and gay men: Implications for social policy and clinical practice. J. GLBT Fam. Stud. 2019, 15, 76-100. [CrossRef]

54. Bostwick, W.; Hequembourg, A. 'Just a little hint': Bisexual-specific microaggressions and their connection to epistemic injustices. Cult. Health Sex. 2014, 16, 488-503. [CrossRef] [PubMed]

55. Vencill, J.A.; Carlson, S.; Iantaffi, A.; Miner, M. Mental health, relationships, and sex: Exploring patterns among bisexual individuals in mixed orientation relationships. Sex. Relatsh. Ther. 2018, 33, 14-33. [CrossRef]

56. Ghabrial, M.A.; Ross, L.E. Representation and erasure of bisexual people of color: A content analysis of quantitative bisexual mental health research. Psychol. Sex. Orientat. Gend. Divers. 2018, 5, 132. [CrossRef]

57. Dyar, C.; Feinstein, B.A.; Schick, V.; Davila, J. Minority stress, sexual identity uncertainty, and partner gender decision making among nonmonosexual individuals. Psychol. Sex. Orientat. Gend. Divers. 2017, 4, 87. [CrossRef]

58. Arena Jr, D.F.; Jones, K.P. To "B" or not to "B": Assessing the disclosure dilemma of bisexual individuals at work. J. Vocat. Behav. 2017, 103, 86-98. [CrossRef]

59. Gonzales, G.; Przedworski, J.; Henning-Smith, C. Comparison of health and health risk factors between lesbian, gay, and bisexual adults and heterosexual adults in the United States: Results from the National Health Interview Survey. JAMA Intern. Med. 2016, 176, 1344-1351. [CrossRef] [PubMed]

60. Oh, H.S. Important significant factors of health-related quality of life(EQ-5D) by age group in Korea based on KNHANES(2014). J. Korean Data Inf. Sci. Soc. 2017, 28, 573-584.

61. Statistics Korea. 2018 Social Survey (Family, Education, Health, Safety and Environment); 2018.11.06.; Statistics Korea: Daejeon, Korea, 2018.

62. Caceres, B.A.; Streed Jr, C.G.; Corliss, H.L.; Lloyd-Jones, D.M.; Matthews, P.A.; Mukherjee, M.; Poteat, T.; Rosendale, N.; Ross, L.M.; Cardiovascular, A.H.A.C.o.; et al. Assessing and addressing cardiovascular health in LGBTQ Adults: A scientific statement from the american heart association. Circulation 2020, 142, e321-e332. [CrossRef] 